\title{
Rechter Populismus in der EU: keine einheitliche Bewegung trotz wachsender Euroskepsis
}

\author{
Florian Hartleb*
}

Populismus ist zu einem Dauerthema in der europäischen Politik geworden, was längst auch in Brüssel, einem der Hauptfeindbilder der rechtspopulistischen Parteien, für Beunruhigung sorgt. Obwohl sich der politische wie intellektuelle Diskurs in Brüssel eher mit Themen wie der Europäischen Nachbarschaftspolitik, aktuell den Entwicklungen im Nahen und Mittleren Osten (,Arabischer Frühling ${ }^{6}$ ) und der eigenen Historie beschäftigt, ist die Frage nach Gefährdungen für das europäische Einigungswerk oben auf der politischen Agenda angekommen - parteiübergreifend und EU-immanent. Unlängst konnte der Verfasser insgesamt fünf ambitionierte und fachlich profunde Konferenzen „zur Gefahr des Populismus“ beobachten. ${ }^{1}$ Die von der Open Society Foundations organisierte Tagung trug gar den Titel: „,We say what you think': Is populism the future of European politics?“", an der unter anderem der namhafte britische Populismusforscher Paul Taggart teilnahm. ${ }^{2}$

Neben der Sorge aller Parteien über das Phänomen gibt es durchaus existente Konfliktlinien oder Missklänge, die den österreichischen Politikwissenschaftler Anton Pelinka von , cleavages ${ }^{6}{ }^{3}$ innerhalb der Europäischen Union sprechen lassen ${ }^{4}-$ die ,cleavage'-Theorie wird bis heute für die Erklärung der gesellschaftlichen Entstehung von Parteiensystemen herangezogen. Pelinkas Auffassung nach gibt es durch tiefe Gräben (,Konfliktlinien`) in den Gesellschaften der Mitgliedstaaten Tendenzen von Euroskeptizismus. Immer noch sei die Europäische Union ein Elitenprojekt, wie sich in den Ablehnungen des Verfassungsvertrags in den Niederlanden und Frankreich ${ }^{5}$ und später des Vertrags von Lissabon im ersten irischen Referendum ${ }^{6}$ zeigte. Es gebe kein europäisches Parteiensystem, vielmehr nationale Parteiensysteme. Widersprüchliche Erwartungen der Mitgliedstaaten zeigen sich in der Außen- und Sicherheitspolitik, mehr noch in der Definition dessen, was eigentlich Identitäts-

* Dr. Florian Hartleb, Research Fellow, Centre for European Studies, Brüssel.

1 Im Europäischen Parlament fanden am 30. und 31. März 2011 sowie 21. Juni 2011 organisiert von den Grünen (unter anderem mit Cas Mudde, der das bislang beste Überblickswerk unter Einschluss Osteuropas verfasst hat; vgl. Cas Mudde: Populist Radical Right Parties in Europe, Cambridge 2007), den Liberalen, den Sozialisten und den Christdemokraten vier Konferenzen zu dem Thema statt. Eine Veranstaltung der Open Society Foundation mit ihrem Initiator, dem Investor George Soros, fand am 7. November 2011, ebenfalls in Brüssel statt. Vgl. Florian Hartleb: Vier Konferenzen von vier verschiedenen Fraktionen (Grüne, Liberale, Christdemokraten und Sozialisten) zum selben Thema mit dem Titel „Neue Gefahr des Populismus“ im Europäischen Parlament (30./31. März 2011 und 21. Juni 2011) in Brüssel, in: Zeitschrift für Parlamentsfragen 2/2011, S. 466-469.

2 Vgl. Paul Taggart: Populism, Buckingham 2000.

3 Seymour M. Lipset/Stein Rokkan: Cleavage Structures, Party Systems and Voter Alignments, in: Seymour M. Lipset/Stein Rokkan (Hrsg.): Party Systems and Voter Alignments: Cross National Perspectives, New York 1967, S. 1-64.

4 Vgl. Anton Pelinka: Bestimmungsfaktoren des Euroskeptizismus, in: Anton Pelinka/Fritz Plasser (Hrsg.): Europäisch Denken und Lehren. Festschrift für Heinrich Neisser, Innsbruck 2007, S. 233-247.

5 Zu den Ursachen des Ausgangs des französischen Referendums siehe Joachim Schild: Ein Sieg der Angst das gescheiterte französische Verfassungsreferendum, in: integration 3/2005, S. 187-200.

6 Zu den Ursachen des Ausgangs des ersten irischen Referendums siehe beispielsweise Jo Leinen/Jan Kreutz: Das irische ,Nein“ zum Vertrag von Lissabon: Optionen für die Lösung der neuen Krise, in: integration 3/ 2008, S. 307-311; Wolfgang Wessels: Die Debatte nach ,Irland': Festhalten an Lissabon, Aufbruch zu Alternativen oder doch Leben mit Nizza?, in: integration 3/2008, S. 312-318; Heinrich Schneider: ,Weiter so! ‘ - oder ganz anders? Die Europapolitik nach dem irischen ,Nein', in: integration 3/2008, S. 318-325. 
kern der Europäischen Union sei. Diese ungelöste Frage wirke sich auf künftige Erweiterungen aus, insbesondere in der Türkeifrage. Die Intensität des Euroskeptizismus ist offenkundig ein mächtiges, einflussreiches Merkmal in der politischen Landschaft der Europäischen Union. Der folgende Beitrag will zuerst die aktuelle Problemlage skizzieren, um dann die Potenziale von Rechtspopulismus und Euroskeptizismus abwägen zu können. Am Ende steht ein Fazit, das Fragen im Umgang mit dem Rechtspopulismus erörtert und künftige Perspektiven bereithält.

\section{Problemlage: Versagen der wirtschaftlichen und politischen Eliten und Erfolg für Euroskeptiker}

Mehr und mehr wird der Rechtspopulismus in die Debatte um die Zukunft der europäischen Integration im weiteren und der Europäischen Union im engeren Sinne einbezogen. Insbesondere die nationalen und europäischen Eliten aus den Reihen der in Westeuropa schrumpfenden sozialdemokratischen und christdemokratisch-konservativen Parteien, im deutschen Kontext als Volksparteien bezeichnet, zeigen sich besorgt, zumal die Krise in einigen der Mitgliedstaaten der Eurozone 2011 für eine tief greifende Diskussion über Stärken und auch offensichtliche Schwächen des europäischen Projekts sorgt. Offenkundig geht es nun in der Eurokrise um die ganze Konstruktion der Europäischen Union in Wirtschaftsfragen und die Finalitätsfrage. Damit sind auch Fragen politischer und sogar kultureller Tragweite impliziert.

Offenkundig gibt es Konstruktionsfehler, wie die Schuldenkrise durch zahlreiche in Not geratene Mitgliedstaaten der Eurozone beweist. Griechenland hätte man gar nicht aufnehmen dürfen, wie trotz der Manipulationen der Statistiken schon zum Zeitpunkt der Zulassung Griechenlands zur dritten Stufe der Wirtschafts- und Währungsunion klar war. ${ }^{7}$ Nun müssen die europäischen wie nationalen Eliten den Bevölkerungen die komplizierte Problematik vermitteln. Das sorgt für Unmut, die Abwahl zahlreicher Regierungen, Chaos auf Regierungsebene (Griechenland, Slowakei mit Neuwahlen und Italien) sowie Protesten auf den Straßen, die sich gegen die Austeritätsmaßnahmen nach zuvor unverantwortlichem Regieren richten.

Der Präsident der Europäischen Kommission, José Manuel Barroso, äußerte in seiner Grundsatzrede zur „Erneuerung Europas - Rede zur Lage der Union 2011“ am 28. September 2011 im Europäischen Parlament in Straßburg seine Sorge: „Populistische Bewegungen stellen die größten Errungenschaften der Europäischen Union in Frage - den Euro, den Binnenmarkt, ja sogar den freien Personenverkehr." 8 Die deutsche Bundeskanzlerin Angela Merkel, durch die Wirtschaftsstärke Deutschlands eine der Schlüsselfiguren im europäischen Krisenmanagement, bemühte die Formel ,Scheitert der Euro, scheitert Europa!“ In ihrer Regierungserklärung im Deutschen Bundestag, wo es um die Zustimmung für den deutschen Anteil am Rettungsschirm ging, versprach sie zugleich, ,er bleibe bei 211 Milliarden Euro".9

Diese Angst der Eliten hat reale Hintergründe, da die Europäische Union nach Meinung vieler Beobachter vor ihrer schwersten Bewährungsprobe steht. Europäische und nationale Eliten befürchten, dass euroskeptische Parteien als Krisengewinnler hervorgehen. Trotz des

7 Berliner Zeitung: Griechenland darf Euro-Zone 2001 beitreten, 19.05.2000.

8 José Manuel Durão Barroso: Erneuerung Europas - Rede zur Lage der Union 2011, 28.09.2011, SPEECH/11/ 607.

9 Regierungserklärung von Bundeskanzlerin Dr. Angela Merkel zum Europäischen Rat und zum Eurogipfel am 26. Oktober 2011 in Brüssel vor dem Deutschen Bundestag am 26. Oktober 2011 in Berlin, in: Bulletin der Bundesregierung Nr. 111-1 vom 26. Oktober 2011. 
fortschreitenden Integrationsprozesses ist das Thema ,Europäische Union“ in der Bevölkerung nicht sehr populär. Blickt man auf die von der Europäischen Kommission veröffentlichten Eurobarometer-Umfragen fällt auf, dass in ganz Europa eine leichte Mehrheit die Europäische Union für eine schlechte Idee hält. ${ }^{10}$ Denkt man an die Rolle der Parteien im intermediären Bereich, die Artikulation, Aggregation, Selektion und Integration von bestehenden Interessen, müsste es logischerweise eine euroskeptische Kraft innerhalb der Europäischen Union geben. Damit ist das Thema durchaus von Relevanz. Die Krise der Eurozone, besonders der Patient, Griechenland" verstärkt diese Furcht. Auch andere Staaten befürchten den Absturz und müssen strenge Austeritätsmaßnahmen einleiten: Spanien, Portugal, Italien (erst unter großem Druck auf Silvio Berlusconi) und auch Frankreich. Bei der jüngsten Parlamentswahl in Finnland am 17. April 2011 wurde gegen die von der Europäischen Union getragene Portugalrettung mobilisiert, sodass die euroskeptische Partei Wahre Finnen fast aus dem Stand heraus auf beinahe 20 Prozent der Stimmen kam. Die nicht-xenophobe Partei stand im Wahlkampf dafür, Hilfszahlungen an die hoch verschuldeten Staaten zu blockieren und den Rettungspakt nachzuverhandeln.

\section{Stärke des Rechtspopulismus in den EU-Mitgliedstaaten}

Seit den frühen 1980er Jahren können neuartige, in erster Linie rechtspopulistische Parteien mit einer Antiestablishment-Haltung, Protestthemen und charismatischen Führungspersönlichkeiten immer wieder Wahlerfolge auf nationaler Ebene erzielen, so zum Beispiel in Frankreich, Österreich, Italien, den Niederlanden, Belgien, der Schweiz und Skandinavien.

Bei den letzten Wahlen in Schweden 2010 und Finnland 2011 konnten dabei erstmals rechtspopulistische Parteien in das Parlament einziehen. Inzwischen kann man von einer ,zweiten Generation“ rechtspopulistischer Parteien sprechen. Am 16. Januar 2011 wurde die Tochter des Parteipatriarchen Jean-Marie Le Pen, Marine Le Pen, in einer Kampfabstimmung zu seiner Nachfolgerin gewählt. In Österreich knüpft Heinz-Christian Strache ${ }^{11}$ an die einstigen Erfolge von Jörg Haider an. ${ }^{12}$ Erfolgreich mit dem Thema ,Antiislam“ ist die niederländische Partei für die Freiheit (Partij voor de Vrijheid, PVV), die 2006 unter anderen von einem aus der Volkspartei für Freiheit und Demokratie (Volkspartij voor Vrijheid en Democratie, VVD) ausgetretenen Mitglied des Parlaments, Geert Wilders, gegründet worden war. Im selben Jahr gewann die Einmitgliedpartei ${ }^{13}$ bei den Parlamentswahlen 5,9 Prozent der Stimmen, 2010 sogar 15,5 Prozent, ein Ergebnis, das viel besser war, als es die Meinungsforscher vorhergesagt hatten. Wilders ließ sofort verlautbaren, dass er - um mitregieren zu können - zu Kompromissen bereit sei (er ließ seinen Widerstand gegen eine Erhöhung des Rentenalters unverzüglich fallen). Bundeskanzlerin Angela Merkel hat die Zusammenarbeit des Christlich-Demokratischen Aufrufs (Christen Democratisch Appèl, CDA), der wie die deutschen Christdemokraten der Europäischen Volkspartei (EVP) angehört, mit Wilders bereits als unvernünftig bezeichnet. Die niederländische Regierungskoali-

10 Vgl. als derzeit aktuellste Eurobarometer-Umfrage: Standard Eurobarometer 75. Die Öffentliche Meinung in der Europäischen Union. Frühjahr 2011 - TNS opinion \& social, August 2011.

11 Siehe beispielsweise zum letzten Europawahlkampf in Österreich Heinrich Schneider: Zwischen ,Policy Determination " und ,Bekenntnisritual'. Anmerkungen zum EP-Wahlkampf und zu seinem Ergebnis in Österreich, in: integration 3/2009, S. 210-230, hier S. 221-223.

12 Vgl. Florian Hartleb: Nach ihrer Etablierung. Rechtspopulistische Parteien in Europa. Begriff - Strategie Wirkung, Zukunftsforum Politik, Sankt-Augustin/Berlin 2011; Florian Hartleb: After their establishment: Right-wing Populist Parties in Europe, Centre for European Studies/Konrad-Adenauer-Stiftung, Brüssel 2011.

13 Allein Wilders ist Mitglied der Partei. 
tion ist mit Wilders' extremen Meinungsäußerungen zum Islam konfrontiert, dieser hat beispielsweise den Koran mit Hitlers „Mein Kampf“ verglichen. In seinen Reden, Kommentaren und Interviews zeigt Wilders eine immer radikaler werdende Variante der Islamphobie. ${ }^{14}$ Diese gründet sich auf eine Vielzahl von Verschwörungstheorien zur bevorstehenden Unterwerfung Europas. Am 11. September 2010 in New York war Wilders einer der wichtigsten Sprecher bei einer Demonstration gegen den Bau eines islamischen Gebetszentrums in unmittelbarer Nachbarschaft des Ground Zero. Im eigenen Land musste sich Wilders von Oktober 2010 an wegen seiner harten Kritik am Islam vor Gericht verantworten, wurde aber im Juni 2011 freigesprochen.

\section{Abbildung 1: Aktueller Erfolg rechtspopulistischer Parteien in Westeuropa (gemäß der letzten Wahlen; geordnet nach den Wahlerfolgen)}

\begin{tabular}{|c|c|c|c|c|}
\hline & & \multicolumn{2}{|c|}{ Letzte nationale Wahl } & \multirow{2}{*}{$\begin{array}{c}\begin{array}{c}\text { Europawahl } \\
2009\end{array} \\
\text { Resultate }^{* *}\end{array}$} \\
\hline Land & Politische Partei & Datum & Resultate* & \\
\hline Norwegen & $\begin{array}{l}\text { Fortschrittspartei } \\
\text { (Fremskrittspartiet - FRP) }\end{array}$ & 14.09.2009 & $22,9 \%$ & - \\
\hline Finnland & $\begin{array}{l}\text { Wahre Finnen } \\
\text { (Perussuomalaiset - PS) }\end{array}$ & 17.04.2011 & $19,0 \%$ & $9,8 \%$ \\
\hline Österreich & $\begin{array}{l}\text { Freiheitliche Partei Österreichs } \\
\text { (FPÖ) }\end{array}$ & 28.09.2008 & $17,5 \%$ & $12,71 \%$ \\
\hline Niederlande & $\begin{array}{l}\text { Freiheitspartei } \\
\text { (Partij voor de Vrijheid - PVV) }\end{array}$ & 09.06 .2010 & $15,5 \%$ & $16,97 \%$ \\
\hline Dänemark & $\begin{array}{l}\text { Dänische Volkspartei } \\
\text { (Dansk Folkeparti - DF) }\end{array}$ & 15.09 .2011 & $12,3 \%$ & $15,3 \%$ \\
\hline Österreich & Bündnis Zukunft Österreich (BZÖ) & 28.09.2008 & $10,7 \%$ & $4,58 \%$ \\
\hline Italien & Lega Nord (LN) & 13.-14.04.2008 & $8,3 \%$ & $10,2 \%$ \\
\hline Belgien & Vlaams Belang (VB) & 13.06 .2010 & $7,7 \%$ & $9,85 \%$ \\
\hline Schweden & $\begin{array}{l}\text { Schwedendemokraten } \\
\text { (Sverigedemokraterna - SD) }\end{array}$ & 19.09 .2010 & $5,7 \%$ & $3,27 \%$ \\
\hline Frankreich & Front National (FN) & 10.06 .2007 & $4,3 \%$ & $6,3 \%$ \\
\hline
\end{tabular}

Quellen: * Wolfram Nordsieck: Parties \& Elections. The database about parliamentary elections and political parties in Europe, abrufbar unter: http://www.parties-and-elections.eu/ (letzter Zugriff: 28.11.2011); ${ }^{* *}$ Europäisches Parlament: Europäische Wahlergebnisse, abrufbar unter: http://www.europarl.europa.eu/parliament/archive/staticDisplay.do?language=DE\&id=213 (letzter Zugriff: 28.11.2011).

Die Auflistung soll freilich keine Homogenität einer eng verwobenen Parteienfamilie widerspiegeln, da die Parteien hinsichtlich ihres Radikalitätsgrades ganz unterschiedlich einzu-

14 Vgl. zum Phänomen Wilders, zu seinen Aussagen und seiner Entwicklung Koen Vossen: Vom konservativen Liberalen zum Nationalpopulisten: Die ideologische Entwicklung des Geert Wilders, in: Friso Wielenga/Florian Hartleb (Hrsg.): Populismus in der modernen Demokratie. Die Niederlande und Deutschland im Vergleich, Münster u.a. 2011, S. 77-104. 
stufen sind. Vlaams Belang, Front National und die Freiheitliche Partei Österreichs unterscheiden sich durch ihre Fremdenfeindlichkeit von den eher moderaten Formationen aus Skandinavien. Es gibt auch Grenzfälle wie die Schweizerische Volkspartei, die hier nicht aufgelistet ist, da sie auch zu den konservativen Parteien gezählt werden kann. Vom Einzelfall hängt auch ab, welche Strategien die Mitte-rechts-Parteien im Umgang mit den rechtspopulistischen Herausforderern anwenden. Alle Optionen wurden bereits praktiziert, wobei aus Sicht der rechtspopulistischen Parteien die Tolerierung einer Minderheitsregierung die attraktivste Variante darstellt. Das Tolerierungsmodell hat Tradition in Skandinavien (Norwegen und Dänemark) und wird derzeit in den Niederlanden durch die PVV von Wilders erprobt. Zur Koalitionsbildung kam es in Italien, zur schnellen Schwächung durch Überforderung in der Regierungsverantwortung in Österreich und den Niederlanden (damals unter Pim Fortuyn). Strikte Ausgrenzungspolitik wird dagegen in Belgien und Frankreich betrieben. Nach dem Systemwechsel nach 1989, aber vor allem im 21. Jahrhundert entfaltet der Populismus auch in Mittel- und Osteuropa seine Wirkung, wie sich auch an konservativen Parteien wie der ungarischen Partei Fidesz unter Viktor Orbán, die mit einer Zweidrittelmehrheit im ungarischen Parlament die derzeit elektoral erfolgreichste Volkspartei in Europa ist, zeigt. ${ }^{15}$

\section{Systemlogik und Begrifflichkeit des Populismus}

Der Terminus Populismus (von lateinisch populus $=$ Volk) ist im Vergleich zu Begriffen wie Liberalismus, Konservatismus oder Sozialismus nicht das Resultat einer historischen Genealogie oder einer geistig-ideengeschichtlichen Fortentwicklung. Oftmals ist der Begriff dabei negativ konnotiert und beinhaltet den Vorwurf, der andere rede dem Volk nach dem Munde und schüre latent vorhandene Ängste und Vorurteile. Positiv gewendet gilt der ,Populist" als jemand, der die Probleme der ,kleinen Leute' versteht, sie artikuliert und der direkt mit dem ,Volk' kommuniziert. Hier zeigt sich die Zwiespältigkeit des Begriffs Populismus. Einerseits verkörpert er allein aufgrund seiner Bedeutung demokratische Ideale. Populismus ist nach dieser Logik ein fester Bestandteil von Demokratie. Andererseits, gemäß dem Suffix ,-ismus', intendiert der Terminus Populismus schon per se eine Übersteigerung, welche sich auch gegen Werte des modernen demokratischen Verfassungsstaats, namentlich gegen Repräsentativkörperschaften und demokratische Entscheidungsprozesse, richten kann. Populismus und Demokratie stehen daher in einem Spannungsverhältnis. ${ }^{16}$

Der Rechtspopulismus umfasst ein Konglomerat aus Strömungen, die an die , einfachen Leute" und nicht an bestimmte Schichten, Klassen, Berufsgruppen oder Interessen appellieren. Sowohl privilegierte Schichten als auch gesellschaftliche Randgruppen dienen ihm als Sündenböcke für soziale Missstände. Hieraus ergeben sich zwei zentrale Dimensionen: ${ }^{17}$

- Die vertikale Dimension als allgemeines Merkmal des Populismus: die Abgrenzung gegen die politische Klasse (Institutionen, Altparteien). Sie kommt in einer Stimmung des ,Wir gegen ,die-da-oben' zum Ausdruck.

- Die horizontale Dimension als spezifisch rechte Variante des Populismus: die Abgrenzung gegen Immigranten, Fremde und Kriminelle. Hier wird ein ,Wir' gegen ,die-da-draußen ‘ propagiert.

15 Vgl. für Ungarn Melani Barlai/Florian Hartleb: Ungarischer Populismus und Rechtsextremismus. Ein Plädoyer für die Einzelfallforschung, in: Südosteuropa Mitteilungen 4/2008, S. 34-51.

16 Vgl. Florian Hartleb: Populismus, in: Martin Hartmann/Claus Offe (Hrsg.): Politische Theorie und Philosophie, München 2011, S. 53-55, hier S. 55.

17 Vgl. Friso Wielenga/Florian Hartleb: Einleitung, in: Friso Wielenga/Florian Hartleb (Hrsg.): Populismus in den Niederlanden und in Deutschland im Vergleich, Münster 2011, S. 7-16. 


\section{Euroskeptizismus als Mobilisierungsthema der Rechtspopulisten}

Trotz des fortschreitenden Integrationsprozesses ist das Thema ,Europäische Union“ in der Bevölkerung nicht sehr populär. ${ }^{18}$ Eine Haltung die durch die Krise der Eurozone noch verstärkt wird, wie der Erfolg der Wahren Finnen bei der jüngsten Parlamentswahl in Finnland zeigt. Euroskeptizismus kann dann zu einem Mobilisierungsthema werden, wenn die Europäische Union, oder besser ein Mitgliedstaat von ihr, in finanzielle Nöte gerät und nach europäischer Solidarität fragt. Derzeit steht insofern viel auf dem Spiel.

Für die Herausbildung einer euroskeptischen Parteienfamilie fehlt es trotz derartiger konjunktureller Gelegenheitsstrukturen dennoch an einem Identitätskern, an Vertrauen und Solidarität untereinander sowie an einer programmatisch-strategischen Agenda, obwohl beträchtliche Teile der Unionsbürger euroskeptisch eingestellt sind. ${ }^{19}$ Euroskeptizismus ist nicht nur auf die rechtspopulistischen Parteien beschränkt, wenn man auf die starke euroskeptische Formation innerhalb der regierenden britischen Konservativen (,Tories“) blickt.

Der Rechtspopulismus bekundet seine Skepsis gegenüber einem zusammenwachsenden Europa. Rechtspopulistische Parteien bedienen die in der Bevölkerung vorhandene Stimmung gegen ein Europa, welches auf Kosten der eigenen nationalen Identität von der Europäischen Union regiert werde. Die Europäische Union beäugen sie gemäß dem Slogan „Europa ja - EU nein!“20 misstrauisch. Von den rechtspopulistischen Parteien gehen keine positiven Visionen oder Impulse für ein geeintes Europa aus, ganz im Gegenteil. Rechtspopulisten warnen vor einem massiven Einschnitt in die nationale Souveränität und Identität durch die Brüsseler Institutionen. Missstände im derzeitigen Institutionengefüge bieten den idealen Anknüpfungspunkt: Der EU-Politik mangelt es tatsächlich an demokratischer Rechenschaftspflicht, auch wenn der Vertrag von Lissabon die Rechte des Europäischen Parlaments stärkte. ${ }^{21}$

Das EU-Thema lässt sich in verschiedenen Variationen populistisch nutzbar machen. Auf diese Weise können Rechtspopulisten die Schwäche der europäischen Außen- und Sicherheitspolitik anprangern und damit in ihrer typischen Schwarz-weiß-Malerei ein christlichabendländisches Bollwerk gegen einen unberechenbaren Islam propagieren. Oder sie prangern den freien Warenverkehr im Binnenmarkt an und machen ihn für die organisierte Kriminalität verantwortlich. Sie bauen darauf, dass es ein gewaltiges Potenzial an antieuropäischen Ressentiments gibt, welches politisch nutzbar ist. Manche rechtspopulistische Parteien verhalten sich gegenüber der Europäischen Union ambivalent, insbesondere in Immigrationsfragen. Populisten, die längerfristig als Partei ,überleben' wollen, werden allem Anschein nach nicht zum Boykott der Europäischen Union aufrufen, sondern vielmehr, eine ökonomische und kulturelle Festung Europa ‘ mit Ressentiments gegen ,die-da-draußen` anpreisen. Im Unterschied zu rechtsextremen Positionen lehnen Rechtspopulisten den europäischen Einigungsprozess nicht ab. Vorrangig kritisieren sie das ,Wie', nicht das ,Ob '. Weite Beachtung hat wohl auch daher die phänomenologische Unterscheidung von ,hartem“ und „weichem“ Euroskeptizismus gefunden, die Paul Taggart und Aleks Szczerbiak 2002 mit Blick auf die neuen osteuropäischen Beitrittskandidaten der Europäischen Union getroffen haben. Die „,weiche“ Form bedeutet die qualifizierte Ablehnung bestimmter Aspekte des In-

18 Vgl. als derzeit aktuellste Eurobarometer-Umfrage: Standard Eurobarometer 75, August 2011.

19 Vgl. genauer Florian Hartleb: A thorn in the side of European elites: The new Euroscepticism, Centre for European Studies, Brüssel 2011.

20 Vgl. ebenda.

21 Vgl. Gerd Strohmeier: Die EU zwischen Legitimität und Effektivität, in: Aus Politik und Zeitgeschichte 10/ 2007, S. 24-30. 
tegrationsprojekts oder der Europäischen Union in seiner beziehungsweise ihrer gegenwärtigen institutionellen Form. Geläufig ist hier das Argument, nationale Interessen stünden dem supranationalen Vertragswerk entgegen. Die „harte“ Form lehnt die ,Idee Europa“ hingegen in ihren Grundsätzen ab, damit folgerichtig auch den Beitritt zu oder die Mitgliedschaft in der Europäischen Union. ${ }^{22}$ Rechtspopulisten sind in der Regel „,weiche“ Euroskeptiker.

\section{Harter Euroskeptizismus als ideologischer Bestandteil von Rechtsextremismus}

Insgesamt zeigen empirische Befunde ${ }^{23}$ von rechtsextremistischen Formationen, dass diese bei Europawahlen meist ein geringeres Wählerpotenzial abrufen können als bei nationalen Wahlen. Besonders die Wahlmodalitäten wirken gerade in kleineren Staaten limitierend. Eine Ausnahme bildet die British National Party, die auf europäischer Ebene vom Verhältniswahlrecht profitierte und 2009 mit 6,3 Prozent erstmals in das Europäische Parlament einzog.

Rechtsextremisten lehnen als „harte“ Euroskeptiker die Idee ,Europa“ aus fundamentalen Gründen ab. So will die Nationaldemokratische Partei Deutschlands (NPD), die weder im Deutschen Bundestag noch im Europäischen Parlament vertreten ist, völlig neue Wege nach Europa beschreiten und die Europäische Union als Symbol der politischen Globalisierung auflösen. Die Europäische Union gilt ihr als Symbol der Fremdbestimmung. ${ }^{24}$

Bei der Europawahl vom Juni 2009 sorgte jedoch eine neue rechtsextremistische Kraft in Ungarn für Furore. Die erst 2004 aus antikommunistisch gesinnten Studierenden gegründete rechtsextremistische Bewegung Jobbik - Jobbik ist ein ungarisches Wortspiel, eine Steigerung sowohl für , gut' wie ,rechts ' - holte aus dem Stand heraus 14,8 Prozent und wurde knapp hinter den Sozialisten drittstärkste Kraft, später auch bei den Parlamentswahlen im Jahr 2010. ${ }^{25}$ Mit Parolen wie „Ungarn gehört den Ungarn“ ist die Partei nicht nur rechtsextremistisch, romafeindlich und antisemitisch, sondern auch hart-euroskeptisch ausgerichtet. Gesicht für die Kampagne der Europawahl war die frühere Frauenrechtlerin ${ }^{26}$ und jetzige fanatische Rechtsextremistin Krisztina Morvai, die auf allen Wahlplakaten zentral präsent war. Morvai, 1963 geboren, äußerte 2010 in der Tageszeitung Die Welt auf die Frage „Sie wurden ins Europaparlament gewählt. Aber offenbar mögen Sie Ihren Job nicht. Oder wie ist es zu erklären, dass Jobbik für Ungarns Austritt aus der EU kämpft?“ Folgendes: „Wir sind nicht unbedingt für den Austritt aus der EU. Aber wir sind gegen die Schaffung eines europäischen Imperiums. Wir sind dagegen, die Nationalstaaten ihrer Entscheidungsbefugnisse zu berauben und den EU-Institutionen zu übertragen. Es gibt keinerlei Kontrolle über die EU-Kommission. Das ist schrecklich und undemokratisch. Ich bin euroskeptisch, aber habe die Hoffnung, dass wir die EU verändern können. Doch wenn das Schlimmste eintritt

22 Vgl. Paul Taggart/Aleks Szczerbiak: Introduction: Opposing Europe? The Politics of Euroscepticism in Europe, in: Paul Taggart/Aleks Szczerbiak (Hrsg.): Opposing Europe? Comparative and Theoretical Perspectives, Band 1, Oxford 2008, S. 1-15. Bezieht man die Diskussion in Deutschland mit ein, hat der Begriff des Euroskeptizismus kaum Eingang gefunden. Manchmal taucht der spezifisch deutsche Terminus der Europamüdigkeit auf. In der einschlägigen Literatur ist zudem der simple Definitionsversuch von Taggart und Szczerbiak beanstandet worden.

23 Vgl. Eckhard Jesse/Tom Thieme: Extremismus in den EU-Staaten im Vergleich, in: Eckhard Jesse/Tom Thieme (Hrsg.): Extremismus in den EU-Staaten, Wiesbaden 2011, S. 431-482.

24 Vgl. Florian Hartleb: Gegen Globalisierung und Demokratie. Die NPD als eine neue soziale Bewegung im europäischen Kontext?, in: Zeitschrift für Parlamentsfragen 1/2009, S. 115-127.

25 Vgl. Melani Barlai/Florian Hartleb: Rechtsextremismus als Posttransformationsphänomen - der Fall Ungarn, in: Totalitarismus und Demokratie 1/2010, S. 83-104.

26 Sie arbeitete von 2003 bis 2006 im Sachverständigenausschuss der Vereinten Nationen und doziert Strafrecht an der staatlichen Eötvös Loránd Universität in Budapest. 
und wir das 2011 auslaufende Moratorium zum Landverkauf nicht neu verhandeln können, dann sollte Ungarn die EU verlassen. Wir dürfen unser Land nicht preisgeben. Die Europäische Union hat Ungarn mehr nötig als wir die EU."27

Die rechtspopulistischen Parteien lassen sich von antidemokratischen, systemfeindlichen Kräften abgrenzen. Eine Diskussion über mögliche Verbindungslinien zum Extremismus, gar zum Terrorismus entbrannte im Sommer 2011. Der allein handelnde norwegische Terrorist Anders Behring Breivik, verantwortlich für eine Bombenexplosion im Osloer Regierungsbezirk und den Tod von insgesamt 69 Menschen am 22. Juli 2011, in der Mehrzahl Jugendliche auf einem Ferienlager der Arbeiterpartei (norske Arbeiderpartiet), war einst Jungfunktionär der Fortschrittspartei (Fremskrittspartiet), die im Vergleich, ohne rassistischen Unterbau als eher moderat einzustufen ist. Der 32-jährige Breivik, der ein Manifest mit über 1.500 Seiten sowie ein You-Tube-Video mit Hetztiraden gegen „Kulturmarxisten“ und Islamisten hinterlassen hat, war aus der Partei ausgetreten, da sie ihm zu moderat schien. Das Manifest trug dabei den Titel „Europa 2083“, der auf die Schlacht in Wien gegen die Türken von 1683 rekurriert („400 Jahre danach“). Obwohl Breivik im Manifest neben vielen anderen, auch auf aus dem Internet kopierte Verweise auf die Erfolge rechtspopulistischer Parteien rekurriert, wäre es sachlich nicht angemessen, eine direkte Verbindungslinie zu ziehen. Breivik muss unter dem Blickwinkel eines isolierten Terroristen betrachtet werden, nicht als Anhänger einer Bewegung.

\section{Eine einigende Kraft? Euroskeptizismus im Europäischen Parlament}

Euroskeptische Formationen können innerhalb der EU-Institutionen und -Organe ihren Einfluss und ihre Position unmittelbar allein im Europäischen Parlament artikulieren. Dort gibt es durch Zersplitterung in unterschiedliche Gruppen und Allianzen mit europafreundlichen Kräften allerdings keine größere euroskeptische Fraktion. Nach der Europawahl von 2009 bleibt ein disparates, höchst unüberschaubares und sich schnell wandelndes Bild. Als euroskeptische Kräfte im Europäischen Parlament nach 2009 können ausgemacht werden:

- Libertas ist als Bewegung aus dem ,Nein` zum Vertrag von Lissabon im ersten Referendum in Irland entstanden, verfügt aber über nur einen Sitz.

- Genuin euroskeptisch ist die Fraktion Europa der Freiheit und Demokratie (EFD), der die britische UKIP-Partei angehört; als Fraktion ist sie klein und heterogen.

- Die Fraktion Europäische Konservative und Reformisten (ECR) wird im Wesentlichen von den britischen Conservatives und der tschechischen Demokratischen Bürgerpartei (Občanská demokratická strana, ODS) sowie der polnischen Partei Recht und Gerechtigkeit (Prawo i Sprawiedliwość, PiS)getragen; sie ist heterogen. ${ }^{28}$

- Da derzeit keine rechtsextremistische Fraktion besteht (ein kurzer Versuch scheiterte zuletzt 2007), gibt es zahlreiche fraktionslose Abgeordnete aus rechtsextremen Parteien, wie der ungarischen Jobbik.

- Daneben existieren fraktionslose nicht-rechtsextremistische Euroskeptiker, wie die österreichische Liste Dr. Martin. ${ }^{29}$

- Partieller Euroskeptizismus findet sich zudem bei der Fraktion „Vereinte Europäische Linke/Nordische Grüne Linke“.

27 Krisztina Morvai: Ungarn muss notfalls aus der EU austreten, in: Die Welt, 12.04.2010.

28 Siehe David Allen: Die EU-Politik der britischen Koalitionsregierung: Distanz vor Pragmatismus, in: integration 3/2011, S. 197-213, hier S. 198-199.

29 Schneider: Zwischen ,Policy Determination“ und ,Bekenntnisritual', 2009, S. 223-225. 
Libertas als genuin europäischer Parteienverbund konnte trotz Wahlteilnahme in mehreren Staaten nur in Frankreich einen Sitz erringen. Als kleinste Fraktion im Parlament formierte sich das „Europa der Freiheit und Demokratie“ (EFD), die als euroskeptisch bezeichnet werden kann. Bei der Europawahl im traditionell euroskeptischen Vereinigten Königreich 2009 wurde die United Kingdom Independence Party (UKIP) mit 16,5 Prozent die zweitstärkste Partei. Neben den Briten gehören der Fraktion Kräfte der regionalistisch, rechtspopulistischen Lega Nord sowie der rechtsextremistischen slowakischen Nationalpartei (Slovenská národná strana, SNS) an, die von 2006 bis 2010 Mitglied einer sozialdemokratisch geführten Koalitionsregierung war. Aber selbst in dieser Fraktion zeigt sich die Heterogenität, wie sich in der geringen Fraktionsdisziplin zeigt. Die EFD liegt bei den geschlossenen Abstimmungen weit unter den Prozentsätzen der anderen Fraktionen. ${ }^{30}$ Zerstrittenheit prägt auch die UKIP nach der für sie so erfolgreichen Europawahl 2009. Schon 2004 hatte sie 16,8 Prozent erzielt; anschließend verließ der Fernsehtalkmaster Robert Kilroy-Silk die UKIP-Gruppe im Europäischen Parlament. Auch nach 2009 kam es zu internen Turbulenzen. Drei der 13 gewählten Mitglieder schieden seit 2009 aus der Fraktion aus (Stand April 2011). UKIP war zwischen 2004 und 2009 Teil der eurokritischen Fraktion „Unabhängigkeit und Demokratie“ (Ind/Dem-Fraktion); diese erzielte auch wegen des Scheiterns von Libertas ein zu schwaches Ergebnis, um nach 2009 eine Fraktion bilden zu können.

Eine weitere euroskeptische Front ist in der Fraktion ECR versammelt. Hier gibt es eine west- und osteuropäische Allianz von Formationen wie den britischen Conservatives und der tschechischen ODS, die nach der Europawahl 2009 aus der EVP auch wegen deren Europafreundlichkeit ausgeschieden sind. ${ }^{31}$ Auch die polnische PiS wurde Mitglied. Ende 2010 waren vier PiS-Abgeordnete an der Gründung der neuen polnischen Partei Polen ist das Wichtigste (Polska Jest Najważniejsza, PJN) beteiligt, die sich eine gemäßigtere konservative Ausrichtung als die PiS gab. Die Mitglieder blieben aber in der Fraktion ECR. Bei der konstituierenden Sitzung des neu gewählten Parlaments am 14. Juli 2009 kam es zu einem Eklat, als der britische ECR-Abgeordnete Edward McMillan-Scott für das Amt eines Parlaments-Vizepräsidenten kandidierte, obwohl die ECR nicht ihn, sondern den Polen Michał Kamiński (damals noch PiS) vorgeschlagen hatte. Durch die demonstrative Unterstützung der anderen Fraktionen wurde McMillan-Scott zum Vizepräsidenten des Parlaments gewählt, ${ }^{32}$ während Kamiński scheiterte.

Zwischen 1999 und 2009 bildete die Union für ein Europa der Nationen eine Fraktion im Europäischen Parlament. Hier waren auch radikal-rechtspopulistische Formationen wie die Dänische Volkspartei (Dansk Folkeparti, DF) und die Lega Nord aktiv, ebenso die nationalpopulistischen polnischen Parteien Selbstverteidigung der Republik Polen (Samoobrona Rzeczpospolitej Polskiej) und Liga der Polnischen Familien (Liga Polskich Rodzin). Nach der Europawahl 2009 hörte die Fraktion auf zu existieren. So wurde die postfaschistische, nationalkonservative italienische Alleanza Nazionale Mitglied im Berlusconi-Bündnis Popolo della Libertà (PdL, Nachfolger der Forza Italia) und damit Teil der Europäischen Volkspartei.

30 Vgl. Group Cohesion Rates auf VoteWatch.eu, abrufbar unter: http://votewatch.eu/cx_european_party_groups. php (letzter Zugriff: 07.09.2011).

31 Vgl. Philip Lynch/Richard Whitaker: A Loveless Marriage: The Conservatives and the European People's Party, in: Parliamentary Affairs 1/2009, S. 31-51.

32 McMillan-Scott wurde daraufhin aus der Fraktion ECR ausgeschlossen und trat im Jahr 2010 zur britischen Partei der Liberal Democrats über und wurde Mitglied der Fraktion Alliance of Liberals and Democrats for Europe (ALDE). 
Rechtsextremisten, die in der Regel ,harte“ Euroskeptiker sind, schafften es nicht, zu einer eigenen Kraft im Europäischen Parlament zu werden. Die nach der zweiten Europawahl im Jahre 1984 gebildete Fraktion der Europäischen Rechten unter Führung des Franzosen Jean-Marie Le Pen war in der Geschichte des Europäischen Parlaments die einzige rechtsextreme Fraktion, die in ihrer Ausgangsstärke über eine volle Legislaturperiode (bis 1989) aufrechterhalten werden konnte. Eine nach der Europawahl 1989 gebildete Fraktion unter Einschluss der deutschen Partei Die Republikaner des damaligen Vorsitzenden Franz Schönhuber scheiterte an der Frage ,Südtirolstatus'.

In den nachfolgenden Legislaturperioden (1994-1999 und 1999-2004) gab es im Europäischen Parlament keine rechtsextreme Fraktion. Der letzte Versuch klappte kurzzeitig 2007, als eine Fraktion namens „Identität, Tradition, Souveränität“ (ITS) entstand - mit dem Versuch, Rechtsextremisten aus West- und Osteuropa zu vereinigen. Ihr gehörten Kräfte wie die österreichische FPÖ, der französische Front National, der belgische Vlaams Belang und die Großrumänenpartei (Partidul România Mare) an. Insbesondere sah man die Chance, durch den EU-Beitritt Rumäniens und Bulgariens nach gescheiterten Versuchen zuvor die rechtlichen Voraussetzungen - 20 Mitglieder des Europäischen Parlaments aus sechs verschiedenen Mitgliedstaaten ${ }^{33}$ - zu erfüllen. Hauptziele waren der Kampf gegen den Vertrag über eine Verfassung für Europa (Verfassungsvertrag), der Kampf gegen Zentralisierungstendenzen und der gegen den möglichen EU-Beitritt der Türkei gerichtete Kampf um den Erhalt nationaler Identität. Die Europäische Union selbst sollte nach dem Willen der Initiatoren den Weg eines Staatenbundes souveräner Nationalstaaten beschreiten.

Wenige Monate nach Formierung, im November 2007, wurde die Fraktion wieder aufgelöst - ein Symptom dafür, dass die europäische Zusammenarbeit von rechtsextremistischen Parteien schwierig ist und sich nur schwer eine praktisch agierende Parteienfamilie herauskristallisieren kann. Die Fraktion fiel unter das Mindesterfordernis an Abgeordneten, die zur Bildung einer Fraktion notwendig sind, nachdem fünf Mitglieder des Europäischen Parlaments der Großrumänenpartei aus Protest gegen die italienische Abgeordnete Alessandra Mussolini (PdL) austraten. Die Rumänen entrüsteten sich über die Äußerungen der Enkelin des ,Duce', Rumänen in Italien zeichne ein krimineller Lebensstil aus. Mussolini hatte Bezug auf den Mord an einer Italienerin genommen, für den ein Roma aus Rumänien verantwortlich gemacht wurde. ${ }^{34}$

Insgesamt ist das Gros der rechtsextremen Abgeordneten im Europäischen Parlament fraktionslos, da nationale Befindlichkeiten eine institutionalisierte Kooperation oft unmöglich machen. Das gilt beispielsweise für die drei Abgeordneten der ungarischen Jobbik.

Euroskeptizismus muss nicht per se ,rechts' ausgerichtet sein. Viele aus der Spitze der Sozialistischen Partei (Parti socialiste) riefen 2005 zu einem ,Nein` im französischen Referendum auf. Die Fraktion Europäische Linke, die mit 35 Mitgliedern (Stand April 2011) als Fraktion sozialistischer und postkommunistischer Parteien als Vereinte Europäische Linke/ Nordische Grüne Linke organisiert ist, stellt das politische und wirtschaftliche System der Europäischen Union auf ihre mangelnde soziale und demokratische Politik hin infrage. Sie verurteilt die aktuelle, ihrer Meinung nach neoliberale Entwicklung der Europäischen Union als unsozial, undemokratisch und damit als gefährlich und fordert eine quasi absolute Umstrukturierung aller Bereiche der Europäischen Union - vom Wirtschaftssystem über die Verteidigungs-, Agrar- und Klimapolitik bis hin zur demokratischen Struktur. ${ }^{35}$ Trotz alle-

33 Derzeit müssen zur Bildung einer Fraktion dieser mindestens 25 Abgeordnete, die in einem Viertel der Mitgliedstaaten gewählt wurden, angehören. Art. 30 Abs. 2 der Geschäftsordnung des Europäischen Parlaments.

34 Vgl. Hartleb: Gegen Globalisierung und Demokratie, 2009.

35 Vgl. Uwe Backes/Patrick Moreau (Hrsg.): Communist and Post-Communist Parties in Europe, Göttingen 2008. 
dem kann man nicht behaupten, dass die Europäische Linke und ihre Fraktion „harte“, unqualifizierte Euroskeptiker sind. Sie scheinen keinerlei Absicht zu haben, dem europäischen Integrationsprozess entgegenzuwirken.

Insgesamt vertritt die Fraktion „Vereinte Europäische Linke/Nordische Grüne Linke“ eine heterogene Position, durchaus analog zu ihren Mitgliedsparteien. So brach in der deutschen Partei Die Linke vor der Europawahl 2009 ein Streit bei der Kandidatenaufstellung aus. Europafreundliche Politiker wie die einstige Spitzenkandidatin zu den Europawahlen 1994, 1999 und 2004, Sylvia-Yvonne Kaufmann, und der einstige Vordenker der Partei, André Brie, wurden nicht in den Listenvorschlag der Partei für die Listenaufstellung zu den Europawahlen 2009 aufgenommen, ${ }^{36}$ da sie entgegen der Parteilinie den Vertrag von Lissabon befürworteten. Im Wahlprogramm schlug sich trotz der Bejahung des europäischen Integrationsprozesses Euroskeptizismus nieder - in Form eines düsteren Bildes der Europäischen Union. In der Konsequenz lehnt die Partei Die Linke jegliche wirtschaftliche und soziale Vertiefung der ihrer Meinung nach neoliberalen Union ab. So stimmte die Bundestagsfraktion auch gegen die Rettungsschirme. Auch die niederländische linkspopulistische Partei Socialistische Partij glaubt, dass der Nationalstaat ,seine Verfügungsgewalt gegen die aufdringliche Europäische Union schützen müsse. “37

\section{Ausblick: Euroskeptizismus im Fokus nationaler Debatten}

Insgesamt wird der Blick auf die Bedeutung eines parteipolitisch diskutierten Euroskeptizismus Veränderungen unterliegen, wie der Fall ,Finnland ' mit dem Erfolg der Wahren Finnen zeigt. Sicherlich ist es nicht ausreichend, eine eurorealistische Position einfach und unberechtigterweise in ein negatives Licht zu rücken. Euroskeptikern kommt immerhin das Verdienst zu, Debatten über Legitimität, Effizienz und das ,Wie“ von Integration angestoßen und im Sinne des actio-reactio-Prinzips und des Agenda-Settings forciert zu haben. ${ }^{38}$ Für die weitere europäische Integration und ihre Verankerung bei den Bürgerinnen und Bürgern ist es essenziell, eine legitime, notwendigerweise zu artikulierende Kritik an den vielen Problemlagen der Europäischen Union - Demokratiedefizit, Kritik an kommenden Erweiterungen wie dem Beitritt der Türkei, kritischer Beitrag zur Überregulierung in einzelnen Politikfeldern wie der Landwirtschaft, Skepsis gegenüber der von den finanzstarken Mitgliedstaaten getragenen Solidarität für auch durch eigene Misswirtschaft in Not geratene Mitgliedstaaten - nicht einfach mit Euroskeptizismus zu vermengen beziehungsweise diesen normativ, zudem pauschal negativ aufzuladen.

Die Krise des Jahres 2011 sorgt dafür, dass die europäischen Eliten in Brüssel offen für ein Mehr an wirtschaftlicher und sozialer Integration eintreten und diese auch forcieren werden. Das funktioniert aber nur, wenn für Europa und die Europäische Union erfolgreich bei der breiten Bevölkerung geworben wird. Der Integrationsprozess wird weiterhin von den Regierungen der Mitgliedstaaten getragen, sodass Euroskeptizismus wohl eher weiterhin als Oppositionsinstrument in den nationalen Parteienwettbewerben genutzt werden wird. Euroskeptizismus ist im europäischen Parteienwettbewerb, auch bedingt durch den begrenzten Einfluss der europäischen Integration auf die nationalen Parteiensysteme keine Mainstreamerscheinung. Für eine euroskeptische Parteienfamilie auf EU-Ebene fehlt es trotz derartiger

36 Stefan Reinecke: Die Dissidentin, in: die tageszeitung, 23.02.2009.

37 Gerrit Voermann: Linkspopulismus im Vergleich. Die niederländische Socialistische Partij (SP) und die deutsche Linke, in: Friso Wielenga/Florian Hartleb (Hrsg.): Populismus in den Niederlanden und in Deutschland im Vergleich, Münster 2011, S. 179-204, hier S. 186.

38 Vgl. Cecile Leconte: Understanding Euroscepticism, Houndsmills 2010, S. 37-42. 
konjunktureller Gelegenheitsstrukturen dennoch an einem Identitätskern, an Vertrauen und Solidarität untereinander sowie an einer programmatisch-strategischen Agenda, obwohl beträchtliche Teile der europäischen Öffentlichkeit euroskeptisch eingestellt sind. Diese Teile können ihren Unmut aber lediglich indirekt, über die ,second-order national elections“39 zum Europäischen Parlament und eine im Vertrag von Lissabon eingeführte, ${ }^{40}$ aber in der Praxis wohl diffizil umsetzbare Bürgerinitiative kundtun. Das heißt, es wird auch künftig zu keiner populistischen oder euroskeptischen Internationalen im Sinne einer Parteiformation kommen. Dem Problem des Rechtspopulismus wird also im nationalen Rahmen zu begegnen sein. Es spricht vieles dafür, dass die Auseinandersetzung um die Zukunft der europäischen Integration und der Europäischen Union stärkeren Einfluss auf die politische Debatte in den Mitgliedstaaten haben wird und haben muss.

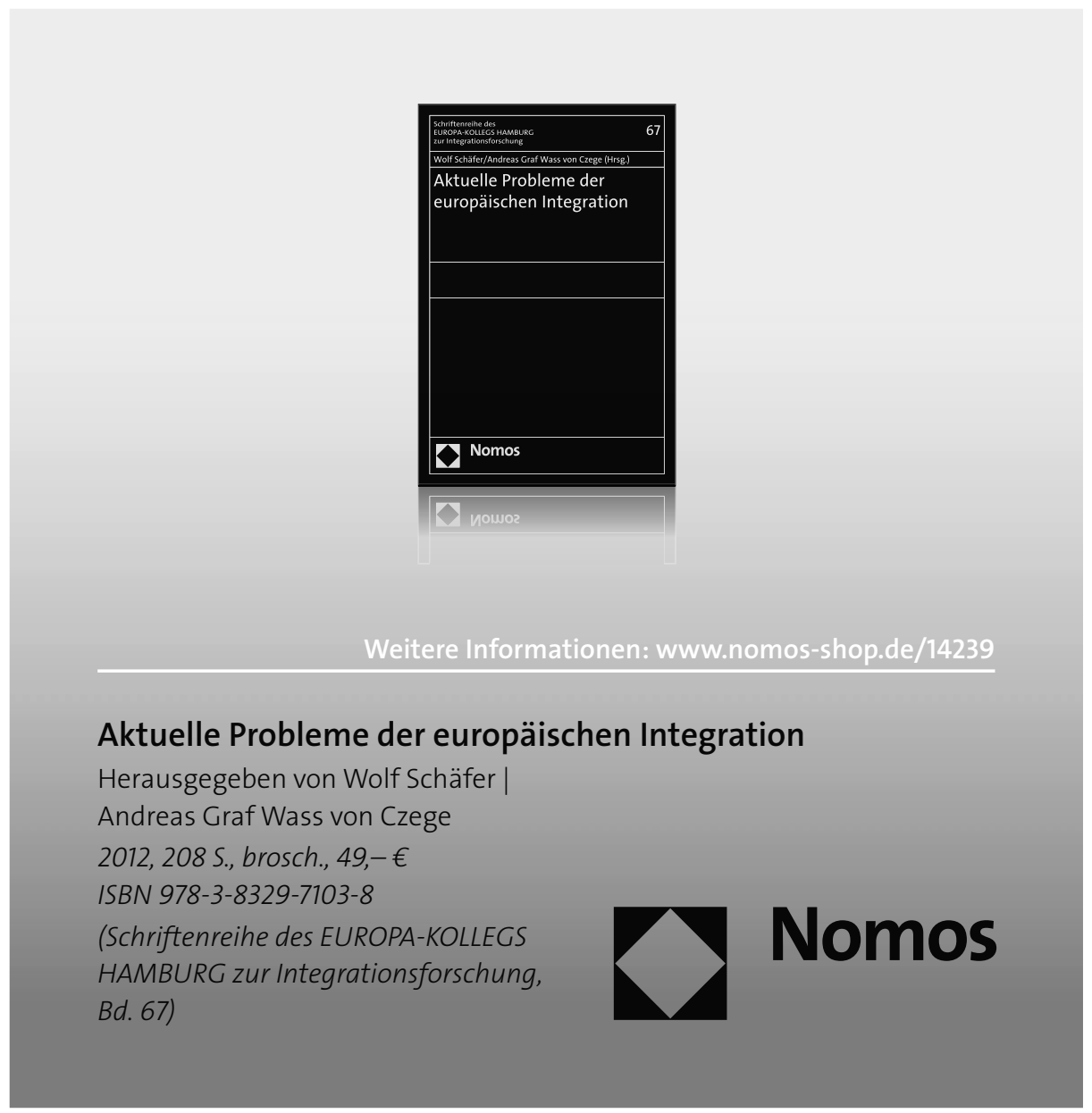

39 Karlheinz Reif/Hermann Schmitt: Nine Second-Order National Elections - A conceptual Framework for the Analysis of European Election Results, in: European Journal of Political Research 1/1980, S. 3-45.

40 Art. 11 Abs. 4 EU-Vertrag. 